\title{
Prostate minimally invasive procedures: complications and normal vs. abnormal findings on multiparametric magnetic resonance imaging (mpMRI)
}

\author{
Thanh-Lan Bui ${ }^{1}$. Justin Glavis-Bloom ${ }^{1}$. Chantal Chahine ${ }^{1} \cdot$ Raj Mehta $^{1} \cdot$ Taylor Wolfe $^{1}$. Param Bhatter ${ }^{1}$. \\ Mark Rupasinghe ${ }^{1} \cdot$ Joseph Carbone ${ }^{1} \cdot$ Masoom A. Haider ${ }^{2} \cdot$ Francesco Giganti $^{3,4}$. Simone Giona ${ }^{5} \cdot$ Aytekin Oto $^{6}$. \\ Grace Lee $^{6} \cdot$ Roozbeh Houshyar ${ }^{1,7}$
}

Received: 13 January 2021 / Revised: 29 March 2021 / Accepted: 19 April 2021 / Published online: 11 May 2021

(c) This is a U.S. government work and not under copyright protection in the U.S.; foreign copyright protection may apply 2021

\begin{abstract}
Minimally invasive alternatives to traditional prostate surgery are increasingly utilized to treat benign prostatic hyperplasia and localized prostate cancer in select patients. Advantages of these treatments over prostatectomy include lower risk of complication, shorter length of hospital stay, and a more favorable safety profile. Multiparametric magnetic resonance imaging (mpMRI) has become a widely accepted imaging modality for evaluation of the prostate gland and provides both anatomical and functional information. As prostate mpMRI and minimally invasive prostate procedure volumes increase, it is important for radiologists to be familiar with normal post-procedure imaging findings and potential complications. This paper reviews the indications, procedural concepts, common post-procedure imaging findings, and potential complications of prostatic artery embolization, prostatic urethral lift, irreversible electroporation, photodynamic therapy, high-intensity focused ultrasound, focal cryotherapy, and focal laser ablation.
\end{abstract}

Keywords Irreversible electroporation · Photodynamic therapy · High-intensity focused ultrasound · Focal cryotherapy · Focal laser ablation · Prostatic artery embolization · Prostatic urethral lift procedure $\cdot$ Benign prostatic hyperplasia .

Prostate cancer

\section{Introduction}

Prostate cancer is one of the most common cancers in the United States [1]. Although roughly 1 in 9 men are diagnosed with prostate cancer in their lifetime, the majority of cases are indolent and associated with a high 5-year survival. Accurate differentiation of nonaggressive versus aggressive cancer types is essential to minimizing risks of overtreatment, particularly in elderly patients with comorbidities who may be poor surgical candidates [2].

Another highly prevalent disease of the prostate is benign prostatic hyperplasia (BPH). It affects most men within their lifetime, with a prevalence of $90 \%$ by the ninth decade of life [3]. As the prostate enlarges, it compresses the prostatic urethra, resulting in difficulties with urination termed

Roozbeh Houshyar

rhoushya@hs.uci.edu

Extended author information available on the last page of the article "lower urinary tract symptoms" (LUTS) and complications including incontinence, urinary retention, and urinary tract infections. Lifestyle modifications and pharmacologic treatments are typically employed as first-line therapy, with prostatectomy traditionally reserved for persistent, debilitating symptoms.

In recent years, a variety of less invasive alternatives to traditional prostate surgery have gained popularity. These minimally invasive procedures aim to mitigate the risks of traditional prostatectomy, which include damage to the neurovascular bundle and urethra and can result in sexual dysfunction and urinary incontinence [4]. These novel procedures include treatments for BPH and localized prostate cancer in select patients. Minimally invasive procedures for localized prostate cancer include irreversible electroporation, photodynamic therapy, high-intensity focused ultrasound, focal cryotherapy, and focal laser ablation; minimally invasive procedures for treating BPH include prostatic artery embolization and prostatic urethral lift. 
To evaluate the prostate, multiparametric magnetic resonance imaging (mpMRI) is increasingly employed and consists of T1-weighted images (T1WI), T2-weighted images (T2WI), diffusion-weighted images (DWI), apparent diffusion coefficient maps (ADC), and dynamic contrastenhanced images (DCE) [5]. It has high sensitivity, specificity, and negative predictive value in detecting prostate cancer [6-8]. Prostate mpMRI images are interpreted and reported using the Prostate Imaging-Reporting and Data System (PIRADSv2), which evaluates lesions utilizing T2WI, DWI, and DCE and produces an overall risk assessment ranging from 1 to 5 [9]. The risk category can be used to determine which lesions should be biopsied and are likely to demonstrate primary clinically significant prostate cancer $[10,11]$. While PI-RADSv2 is useful to detect primary clinically significant prostate cancer, it is not designed to plan treatment. Additionally, it is not appropriate in the post-treatment setting, as the criteria for the detection of recurrent prostate cancer after minimally invasive procedures are likely distinct from PI-RADSv2 criteria.

As minimally invasive procedure and prostate mpMRI volumes continue to increase, radiologists must be familiar with the common procedures, complications, and expected post-procedure appearance of the prostate. These procedures can distort the prostate appearance and may introduce MRI artifacts, which can increase the risk for misinterpretation. This paper offers a review of contemporary minimally invasive prostate procedures for $\mathrm{BPH}$ and localized prostate cancer, associated complications, and normal post-procedure imaging findings.

\section{Minimally invasive procedures for BPH}

\section{Prostatic artery embolization}

Background Prostatic artery embolization (PAE) was approved by the United States Food and Drug Administration (FDA) in 2017 for the treatment of LUTS secondary to $\mathrm{BPH}$ [12]. It is currently indicated for patients with special risks for surgery, sexually active men who wish to avoid the risk of retrograde ejaculation, patients with a prostate volume greater than $65 \mathrm{~mL}$, those with recurrent bleeding secondary to $\mathrm{BPH}$, or patients with a permanent bladder catheter.

Procedure Under local anesthesia and moderate sedation, arterial access is achieved to the common femoral artery [12]. After evaluation of the iliac and prostatic arterial anatomy by angiography and often with confirmation of prostate perfusion by computed tomography (CT), the angiographic endpoint for embolization is determined. Embolization of the prostatic artery utilizing $100-300$ and/or $300-500 \mu \mathrm{m}$ microsphere particles causes ischemic necrosis in the prostate, leading to a reduction in prostate size and LUTS.

Post-procedure imaging (Fig. 1) After PAE, infarcts on the embolized side(s) are characterized by initial hyperintensity on T1WI, which is greatest 1-3 months after the procedure, and hypointensity on T2WI [13, 14]. Over time, these infarcts decrease in size and become isointense on both sequences. They are typically not seen on these sequences at the 12-month follow-up. On DWI, the infarcts demonstrate

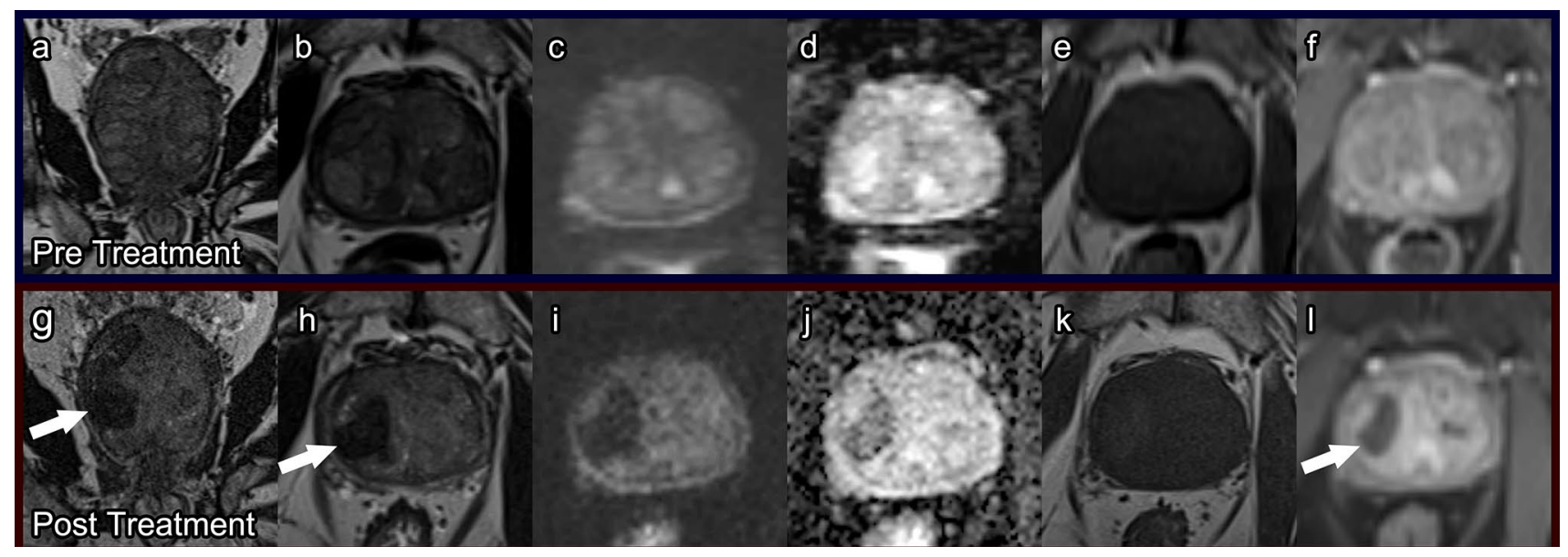

Fig. 1 Prostatic artery embolization. Pre-treatment prostate MRI: Coronal T2 (a), axial T2 (b), axial DWI (c), ADC (d), pre-contrast $\mathrm{T} 1$ (e), and post-contrast T1 (f) demonstrate prostatomegaly with findings of benign prostatic hyperplasia, without diffusion restriction and with normal enhancement. Post-PAE prostate MRI at 4 months: Coronal T2 (g), axial T2 (h), axial DWI (i), ADC (j), precontrast $\mathrm{T} 1(\mathbf{k})$, and post-contrast $\mathrm{T} 1$ (l) demonstrate $\mathrm{T} 2$ dark areas of chronic ischemia with hemosiderin deposition (arrows) no significant diffusion restriction (i), and new hypoenhancement associated with ischemic tissue (arrow, l) 
decreased signal intensity starting at 1 -month post procedure. This decrease in signal intensity corresponds with prostatic softening and is even more evident at 12 months post procedure, which is seen as a sign of treatment success [14]. Twelve months is also when a statistically significant decrease in prostate volumes is typically seen, with volumes decreasing approximately $20-40 \%$ [13-15].

While pre- and post-procedure mpMRI are part of many PAE protocols, there is no consensus on follow-up imaging intervals for monitoring clinical success. Some studies have suggested repeating mpMRI as soon as 1-month post procedure, while others recommend imaging to begin at the 3-month follow-up [13, 15]. Additionally, some studies recommend mpMRI at multiple time points, while others believe that a one-time post-procedure mpMRI demonstrating the above changes is adequate. Of note, some clinicians opt to use ultrasound only post-PAE to measure the decrease in prostate volume. Additionally, in the absence of post-procedure symptoms, some clinicians might argue that postprocedure imaging is unnecessary.

Complications Major complication rates for PAE are reported to be between 0.1 and $0.4 \%$ [16]. The most common complication of PAE is cystic transformation within an area of infarct, which is seen as high signal intensity on T2WI and low signal intensity on T1WI, typically at 12 months post procedure [17]. Another common complication, usually seen within $72 \mathrm{~h}$ of PAE, is "post-embolization syndrome" with symptoms including pain, nausea, vomiting, mild fever, and dysuria [15]. Patients may also experience bladder ischemia, sometimes requiring additional surgery, and persistent perineal pain. Minor complications include dysuria, acute urinary retention, hematospermia, minimal rectal bleeding, and urinary tract infections.

\section{Prostatic urethral lift}

Background The prostatic urethral lift procedure, commonly performed with a UroLift ${ }^{\circledR}$ device (NeoTract-Teleflex, Pleasanton, CA, USA), was first introduced as a feasible treatment for lateral lobe enlargement BPH in 2011 and received FDA approval in 2013 [18]. Because it has reduced rates of procedure-related sexual dysfunction and shorter length of hospital stay, it is an ideal option for men who are sexually active; those who do not want to take lifelong medication; men who have failed medical management; or those who do not want cavitating surgery. Patient eligibility depends on prostate volume and median lobe size $[19,20]$. If the median lobe is too large and obstructing, a lateral lobe prostatic urethral lift procedure is contraindicated.

Procedure The prostatic urethral lift device is delivered under cystoscopic guidance and placed at least $1.5 \mathrm{~cm}$ distal to the bladder neck, near the anterolateral prostate area [18, 19]. It must be angled such that when the needle is deployed, its path will be parallel to the bladder neck. Once in place, the device is deployed and delivers a prosthesis that retracts the lateral lobes of the prostate, which in turn increases flow through the urethra. Flow is assessed cystoscopically, and additional devices can be implanted anterior to the verumontanum as needed to keep the urethra open. After all the devices are deployed and adequate flow is confirmed cystoscopically, the procedure is complete.

Post-procedure imaging (Fig. 2) The prostatic urethral lift prosthesis that is implanted into the patient is composed of a stainless-steel tab and a nitinol tab, which are bridged by suture [21]. The nitinol portion does not show up on imaging. However, the stainless-steel urethral part of the prostatic urethral lift device can generate a $10-15 \mathrm{~mm}$ susceptibility artifact with peripheral hyperintensity. This artifact becomes more prominent on DWI and ADC images and can obscure evaluation of the transition zone, and in some cases the peripheral base (Fig. 2).

Obscurement of the transition zone can make interpretation of imaging for prostate cancer more challenging [22]. Therefore, prior to prostatic urethral lift, patients should be made aware of this limitation, especially if they are high risk for prostate cancer. Additionally, if there is any evidence suggesting that prostate cancer may be developing in a patient who has had a prostatic urethral lift, it is essential for clinicians to include the prostate anterior zone during ultrasound-guided biopsies [21].

Complications Overall complication rates of prostatic urethral lift are low $[19,20]$. The most reported adverse events include dysuria, hematuria, urgency, and pelvic pain. However, most of these complications are mild and self-limiting.

\section{Minimally invasive procedures for localized prostate cancer}

Minimally invasive procedures for treating localized prostate cancer include high-intensity focused ultrasound (HIFU), cryotherapy, irreversible electroporation (IRE), focal laser ablation (FLA), and photodynamic therapy (PDT). These procedures are highly effective and all eventually lead to fibrosis of the treatment area. As such, on delayed mpMRI imaging, post-treatment prostates are expected to have a similar appearance irrespective of the procedure performed - the treated area of the prostate will have T2 dark signal [23-27]. However, short-term post-treatment imaging changes vary based on the procedure. 


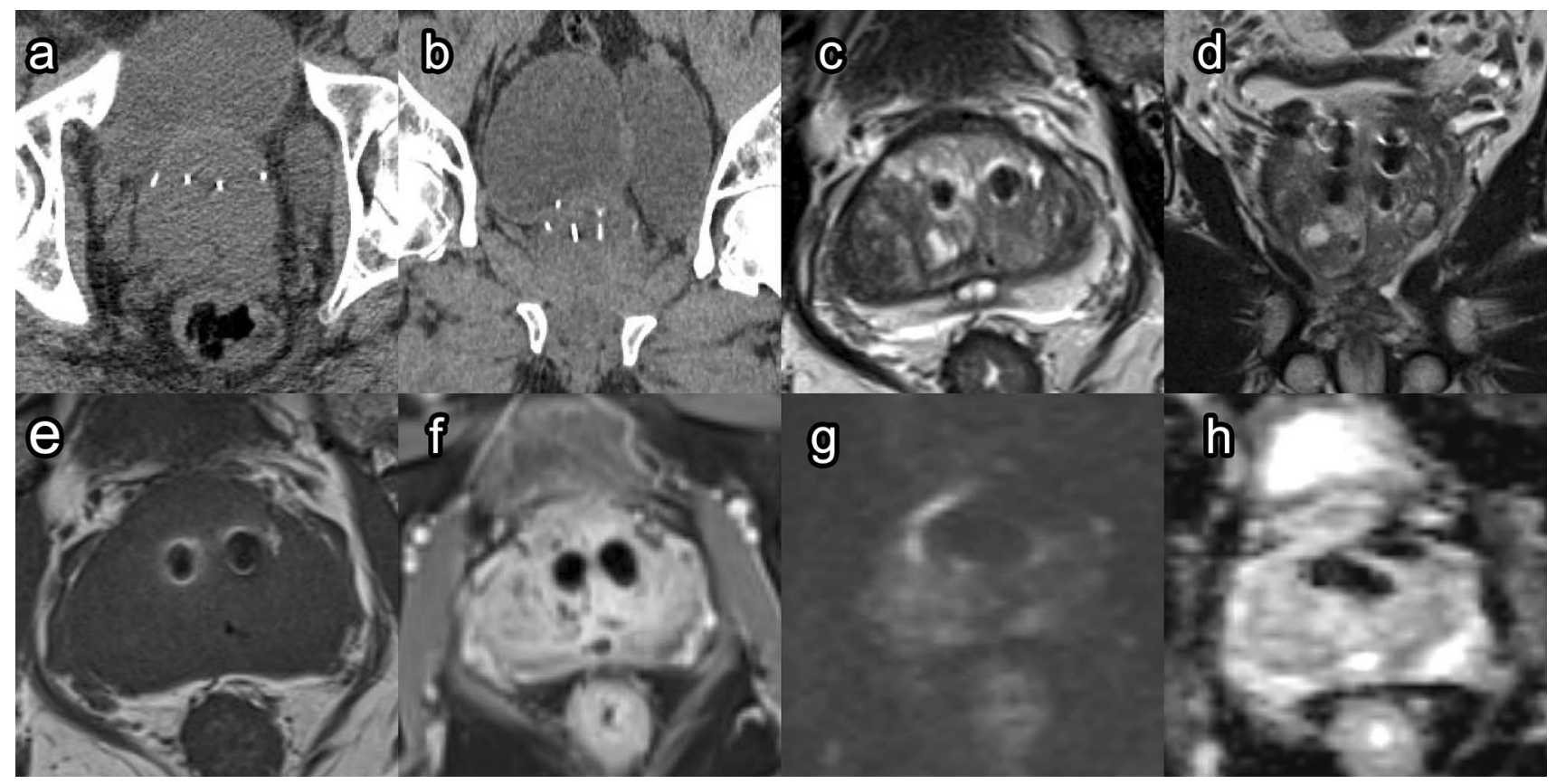

Fig. 2 Prostatic urethral lift. Post-prostatic urethral lift prostate CT: Axial (a) and coronal (b) images of a patient with Urolift ${ }^{\circledR}$ implant. Metallic attenuation objects are noted in appropriate position along the bilateral median lobes. Post-prostatic urethral lift prostate MRI: Axial T2 (c), coronal T2 (d), pre-contrast T1 (e), and post-contrast T1

\section{High-intensity focused ultrasound}

Background HIFU was first introduced as a minimally invasive treatment for prostate cancer in the 1990s and became FDA approved for prostate tissue destruction in 2015 [28]. The procedure is performed for patients with low- and intermediate-risk localized prostate cancer; patients requiring salvage therapy for recurrent prostate cancer after radical prostatectomy, hormone ablation, or radiation therapy; patients with advanced prostate cancer for neoadjuvant debulking; and patients with castration-resistant prostate cancer [29].

Procedure Under general or spinal anesthesia, a HIFU probe is inserted transrectally [28]. The target lesion(s) are visualized and mapped with ultrasound, often utilizing preprocedural MRI fusion guidance. A series of pulsed highenergy sound waves are delivered through the probe to the targeted area, which rapidly raises the target tissue's temperature and promotes cell death. The probe is then repositioned, and the procedure is repeated at other target sites as needed. HIFU can be performed using ultrasound targeting or MRI in-bore targeting.

Post-procedure imaging (Fig. 3) Immediately after HIFU, it is difficult to distinguish the peripheral and transition zones (f) images demonstrate a metal susceptibility artifact causing a signal void with peripheral hyperintensity in the region of the implant. DWI at B1400 (g) and ADC (h) images demonstrate exaggerated signal void in the region of the implant

due to complete loss of zonal anatomy [30]. One to three months post procedure, a "double ring" sign (heterogeneous T2-hypointense treatment zone with a surrounding hyperintense ring) can be seen, which is unique to post-HIFU imaging [26]. The amount of enhancing tissue at this time has been found to correlate with prostate volume at 6 months, PSA level nadir, and biopsy evidence of residual cancer. Therefore, it can be an early indicator of treatment success. The "double ring" sign typically disappears after 6 months. At 6 months, the post-treatment prostate shows low T2 signal intensity with poorer definition of the prostate capsule (Fig. 3). Prostate volume by this time will have decreased 40-60\%.

Formal timelines do not exist for follow-up mpMRI after HIFU. However, based on the expected post-procedure imaging findings, it has been proposed to obtain repeat mpMRI at 6-12 months [26]. Cancer recurrence can be evident as early as 6 months post treatment and is seen as a hypointense nodular lesion on T2WI with restricted diffusion on DWI and rapid wash-in and wash-out of contrast on DCE (Fig. 4) [31]. Cancer recurrence and fibrosis both have low signal on $\mathrm{ADC}$ and T2; however, recurrence demonstrates high signal on DWI and early enhancement while fibrosis may have late enhancement. DCE sequences are most useful in distinguishing recurrence from post-procedure fibrosis [32]. 


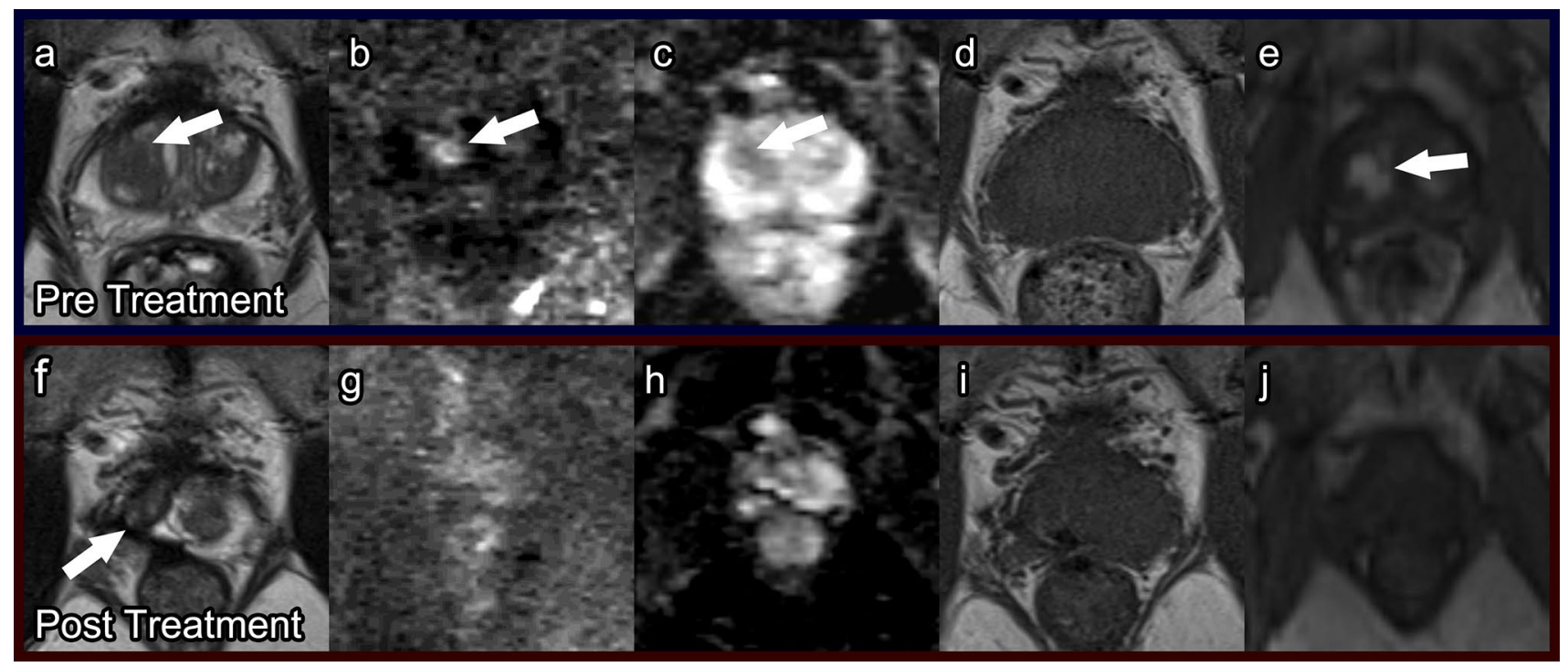

Fig. 3 High-intensity focused ultrasound. Pre-treatment prostate MRI: Axial T2 (a), DWI (b), ADC (c), pre-contrast T1 (d), and postcontrast T1 (e) demonstrate a PI-RADS 5 lesion (arrows) in the right mid-transition zone. Biopsied as Gleason score $4+3$ prostate cancer.
Post-HIFU prostate MRI at 1 year: Axial T2 (f), DWI (g), ADC (h), pre-contrast $\mathrm{T} 1$ (i), and post-contrast $\mathrm{T} 1$ (j) demonstrate linear $\mathrm{T} 2$ dark scarring (arrow) and prostatic atrophy without restricted diffusion or hyperenhancement

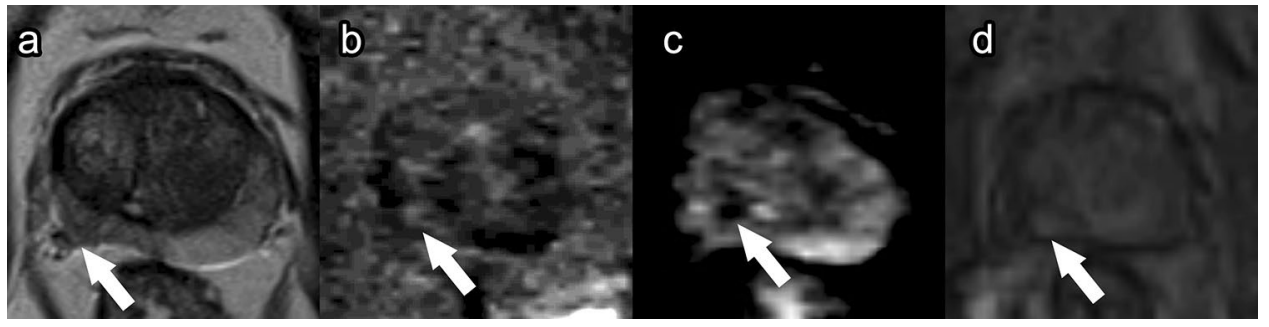

Fig. 4 Recurrence in a patient after high-intensity focused ultrasound. Post-treatment prostate MRI: Axial T2 (a), DWI (b), ADC (c), and post contrast $\mathrm{T} 1$ (d) demonstrate a right posterolateral peripheral zone lesion with heterogeneous T2 dark signal, restricted diffusion and early arterial hyperenhancement (arrows). Biopsied as Gleason 3 +4 recurrent prostate cancer with perineurial invasion

prostatectomy or radiotherapy. When performed for curative purposes, whole gland cryotherapy should be done in patients with T1-T3 tumors without metastatic disease [34]. Prostate size is also a factor taken into consideration, as cryotherapy is more difficult to perform on large prostates (size greater than $60 \mathrm{~g}$ ). Focal cryotherapy can also be done, but no current guidelines exist for its use.

Procedure Under general anesthesia, cryoneedles are inserted into the prostate through either a transrectal or transperineal approach under ultrasound or MR guidance [35]. A urethral warming catheter and thermal sensor are positioned between the anterior rectal wall and prostate capsule to monitor and protect adjacent structures. The cryoneedles are then cooled with argon gas until the target area reaches a temperature of $-40{ }^{\circ} \mathrm{C}$ to $-80^{\circ} \mathrm{C}$. The target area is thawed and refrozen several times, causing cell death and 
promoting coagulative necrosis. The resultant formation of ice crystals and an ice ball distorts and destroys local tissue architecture.

Post-procedure imaging (Fig. 5) In the time period closely following cryotherapy, imaging findings are distinctive due to the repetitive freezing and thawing of prostate tissue. During cryotherapy, the ice ball produces a strong T1 signal void with high-intensity rim [27]. After the procedure, DCE sequences consistently show an enhancing rim around a nonenhancing area. T1WI shows heterogeneous enhancement intermixed with areas of necrosis, and a diffusely hypointense T2 signal is observed within the peripheral zone with impeded diffusion on DWI (Fig. 5). Two months after treatment, thickening of the prostate capsule, rectal wall, and urethra can be seen along with a decrease in prostate volume [27]. When cryotherapy is used to destroy the whole prostate, imaging is often not useful after the procedure to detect recurrence [32]. Frequently, the damaged zone is too large, and any untreated prostate or cancerous tissue is small (less than $5 \mathrm{~mm}$ ) and irregularly shaped, making it challenging to detect on mpMRI. Often times, cryotherapy is used for focal treatment, and the damaged gland is limited by laterality of treatment side. Post-treatment surveillance for local recurrence with mpMRI is best appreciated on DCE sequences [30].

Complications Erectile dysfunction is almost an expected outcome after whole gland cryosurgery because the neurovasculature cannot be preserved [33]. This is especially common with posterior cryotherapy. Therefore, some clinicians perform cryotherapy on anterior lesions and opt for another procedure, such as HIFU, for posterior lesions. Other common complications include damage to the urethra, urinary incontinence, irritative urinary symptoms, and urinary obstruction. Urethrorectal fistula is an uncommon adverse event $(<1 \%)$ [35].

\section{Irreversible electroporation}

Background IRE induces programmed cell death and apoptosis using very short and strong pulsed electric fields [36]. It gained FDA approval in 2019 and is performed in localized low- to intermediate-risk prostate cancer. It has been used for treatment of high-risk prostate cancers (mostly in Europe).

Procedure Under general anesthesia, a transurethral urinary catheter is inserted [36]. Monopolar electrodes are then placed transperineally and positioned around target lesions using MRI-TRUS fusion technique. Correct electrode position and inter-electrode distances are confirmed. IRE pulses are emitted with frequencies appropriate to specific protocols.

Post-procedure imaging (Fig. 6) Immediately post IRE, the prostate volume increases significantly, which is thought to be due to necrosis [37]. At 24-72 $\mathrm{h}$ after the procedure, the ablation zone is heterogeneously or homogenously hypoattenuating with hyperattenuating margins on T1WI, possi-

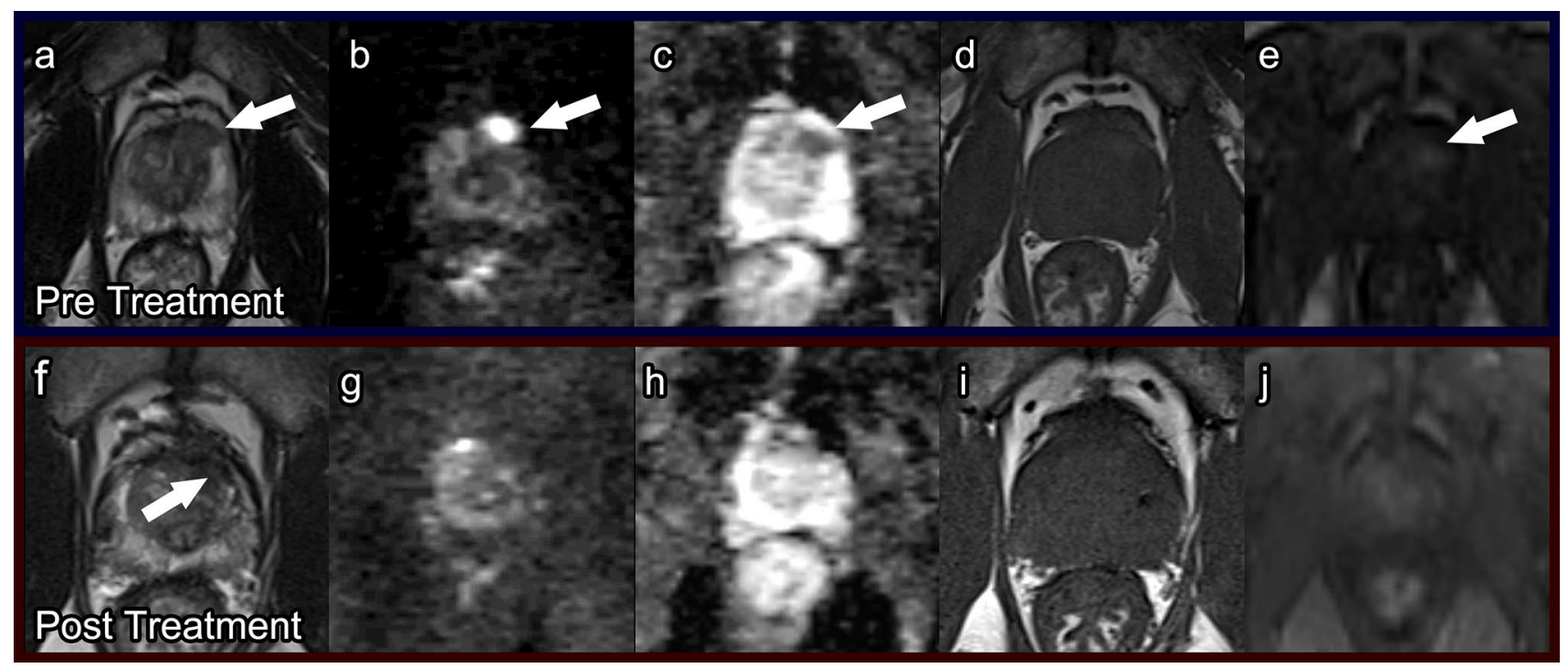

Fig. 5 Cryotherapy. Pre-treatment prostate MRI: Axial T2 (a), DWI (b), ADC (c), pre-contrast T1 (d), and post-contrast T1 (e) demonstrate a PI-RADS 5 lesion (arrows) in the left mid-anterolateral peripheral zone. Biopsied as Gleason score $3+4$ prostate cancer.
Post-cryoablation prostate MRI at 1 year: Axial T2 (f), DWI (g), ADC (h), pre-contrast T1 (i), and post-contrast T1 (j) demonstrate ovoid T2 dark scarring (arrow), mild prostatic atrophy without restricted diffusion or hyperenhancement 
Fig. 6 Irreversible electroporation. Pre-treatment prostate MRI: Axial T2 (a), DWI (b) and post-contrast $\mathrm{T} 1$ (c) demonstrate an anterior left mid-gland lesion (arrows). Biopsied as Gleason score $3+4$ prostate cancer. Post-IRE prostate MRI at 7 days: DCE (d), T2 (e), DWI (f), and pre-contrast $\mathrm{T} 1$ (g) show a post-procedure necrotic area (asterisk) and heterogeneous decreased T2 signal. Post-IRE prostate MRI at 3 years: T2 (h) and DCE (i) indicate residual fibrosis (arrowheads), with no signs of recurrence at the treatment site

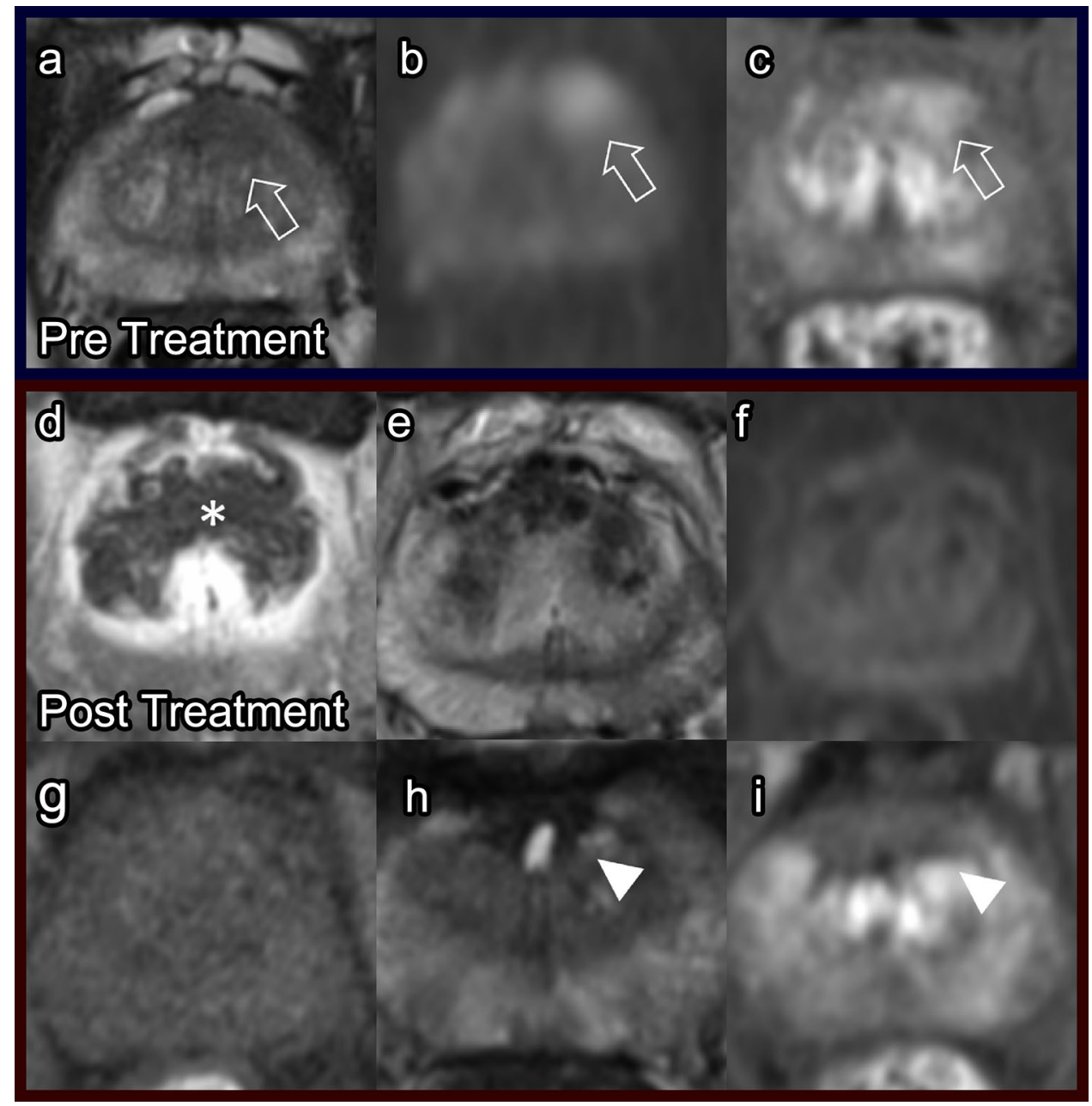

bly due to reactive hyperemia and/or sustained vascularization [38]. This appearance is characteristic of IRE. At 1 month, post-contrast sequences demonstrate areas of nonenhancement, and heterogeneous signal intensity is seen on T2WI [30]. Additionally, areas of low T2 signaling can be observed, which represent fibrosis (Fig. 6) [25]. By 6-12 months, the prostate volume has decreased considerably.

Studies suggest that post-procedure follow-up imaging after IRE should include a repeat mpMRI at 6-12 months [39]. Subsequent mpMRI can be obtained depending on patient-specific factors. Residual or recurrent disease demonstrates low T2 signal, asymmetrical enhancement on DCE, and high signaling on DWI with restricted diffusion on ADC [25]. DCE sequences are the most sensitive for detecting recurrence [30].

Complications Complications are relatively uncommon with IRE, but the most common adverse events include urinary retention and dysuria [37]. They occur more frequently in patients with large prostates or when a large area of prostate was treated. Notably, IRE preserves collagenous and protein or lipid-based structures, such as vascula- ture, and ductal networks, such as the urethra. A large retrospective European study of 429 patients who underwent IRE reported rates of $0 \%$ for urinary incontinence and only $11.3 \%$ for erectile dysfunction [36].

\section{Focal laser ablation (FLA)}

Background The use of FLA is still being investigated with ongoing studies, and no guidelines exist for its use [40]. Larger randomized control trials have yet to be conducted. FLA is currently recommended in select outpatients with low- to intermediate-risk prostate cancer with Gleason Score $\leq 4+3$.

Procedure Under direct MR guidance, a laser fiber is inserted either transperineally or transrectally through a grid towards the target lesion $[24,41]$. Once the correct position is confirmed, a laser, typically potassium-titanyl-phosphate or holmium, is activated for 1 to 2 min using $6-25 \mathrm{~W}$ to heat the target lesion to temperatures as high as $60{ }^{\circ} \mathrm{C}$. This thermally destroys the lesion. Temperature is usually monitored in the neurovascular bundle to help prevent neurovascular 
bundle damage. Repositioning occurs as needed to maximize treatment area. A repeat T2-weighted image is then re-obtained to confirm target lesion cooling. While FLA is typically performed in-bore, studies have demonstrated that it is safe and feasible in the clinic setting also [42].

Post-procedure imaging (Fig. 7) The treatment area after FLA is better defined compared to after other focal therapies because of the targeted nature of the procedure. Immediately after FLA, post-contrast sequences typically show nonenhancing lesions, which decrease in size at 3 months post treatment and disappear by 1 year [24]. DWI shows restricted diffusion, which resolves by 6 months after FLA (Fig. 7) [30]. T2WI demonstrates patchy, low signal intensity within the treatment area at 3-month follow-up, which is especially apparent by 1 year [24].

There is no standard time interval for repeat mpMRI after FLA. However, one study suggested at least one repeat mpMRI 6-12 months post procedure with periodic serial mpMRI performed after depending on patient characteristics [39]. Others have advised waiting until 12 months when post-treatment changes typically settle [30]. Recurrence can be seen as enhancing tissue within an area of scarring.

Complications Overall complications rates are low in FLA [41]. However, the procedure can damage adjacent structures, such as the urethra or neurovasculature. Damage to the rectal wall during fiber positioning may occur if using a transrectal approach. The most commonly reported com- plication is perineal discomfort. No changes in urinary or sexual functions were reported in Phase I trials [41].

\section{Photodynamic therapy}

Background PDT was first introduced in the 1990s as a treatment for low- to intermediate-risk prostate cancer using TRUS [43]. Since then, several generations of photosensitizers for PDT have been developed, which are under evaluation [44].

Procedure A 10-min intravenous injection of a photosensitizing agent occurs prior to PDT [45]. This agent is then activated by optical fibers, which are strategically placed with TRUS using MRI fusion guidance [23, 45]. The activation of the photosensitizing agent creates free radicals that target blood vessels and tumor neovasculature, resulting in tissue devascularization. This may have some selectivity for tumor.

Post-procedure imaging (Fig. 8) The amount of tissue edema and necrosis typically peaks around 1 week after PDT [30] and is observed on T1WI as increased signal intensity and on T2WI as the same or decreased signal intensity [23]. T1WI is considered to be more useful than T2WI due to ongoing coagulative necrosis and hemorrhage [30]. Interestingly, because of fiber placement, the borders of the treatment area are often irregular with projections of enhancing viable tissue interspersed with nonenhancing necrotic tissue, which is specific to post-PDT imaging (Fig. 8). Six months after

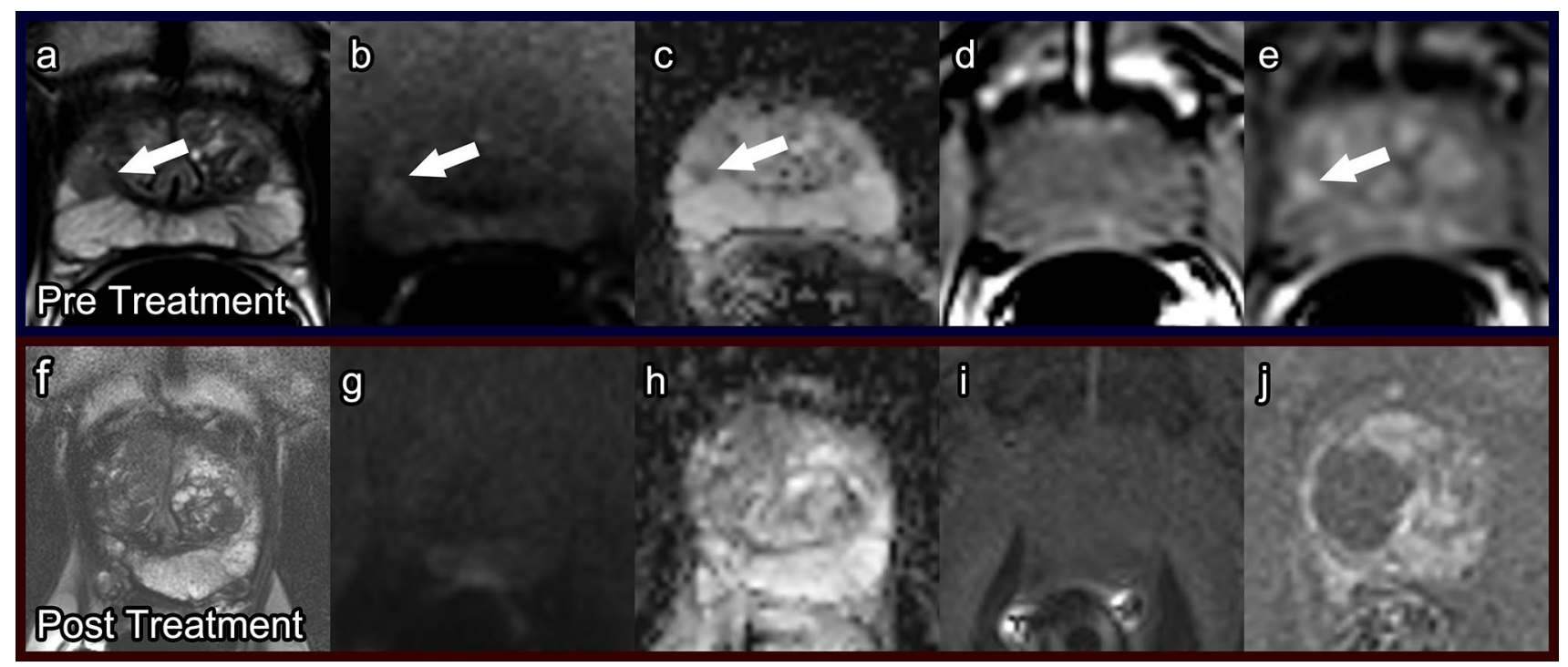

Fig. 7 Focal laser ablation. Pre-treatment prostate MRI: Axial T2 (a), DWI (b), ADC (c), pre-contrast T1 (d), and post-contrast T1 (e) demonstrate a lesion at the right lateral mid-gland to apex peripheral zone (arrows). Post-focal laser ablation prostate MRI at 1 year: Axial T2 (f), DWI (g), ADC (h), pre-contrast T1 (i), and post-contrast T1 (j) demonstrate T2 hypointensity, atrophy, and retraction, with hypoenhancement in the ablated right lateral gland 
Fig. 8 Photodynamic therapy. Pre-treatment prostate MRI in a patient with history of external beam radiation therapy for prostate cancer: T2 (a), ADC (b), and DCE (c) demonstrate heterogeneous prostate enhancement, diffuse restricted diffusion, and heterogeneous T2 dark signal. Post-photodynamic therapy prostate MRI at 7 days: T2 (d), ADC (e), and DCE (f) show post-treatment effect best on the post-contrast imaging with areas of nonenhancement
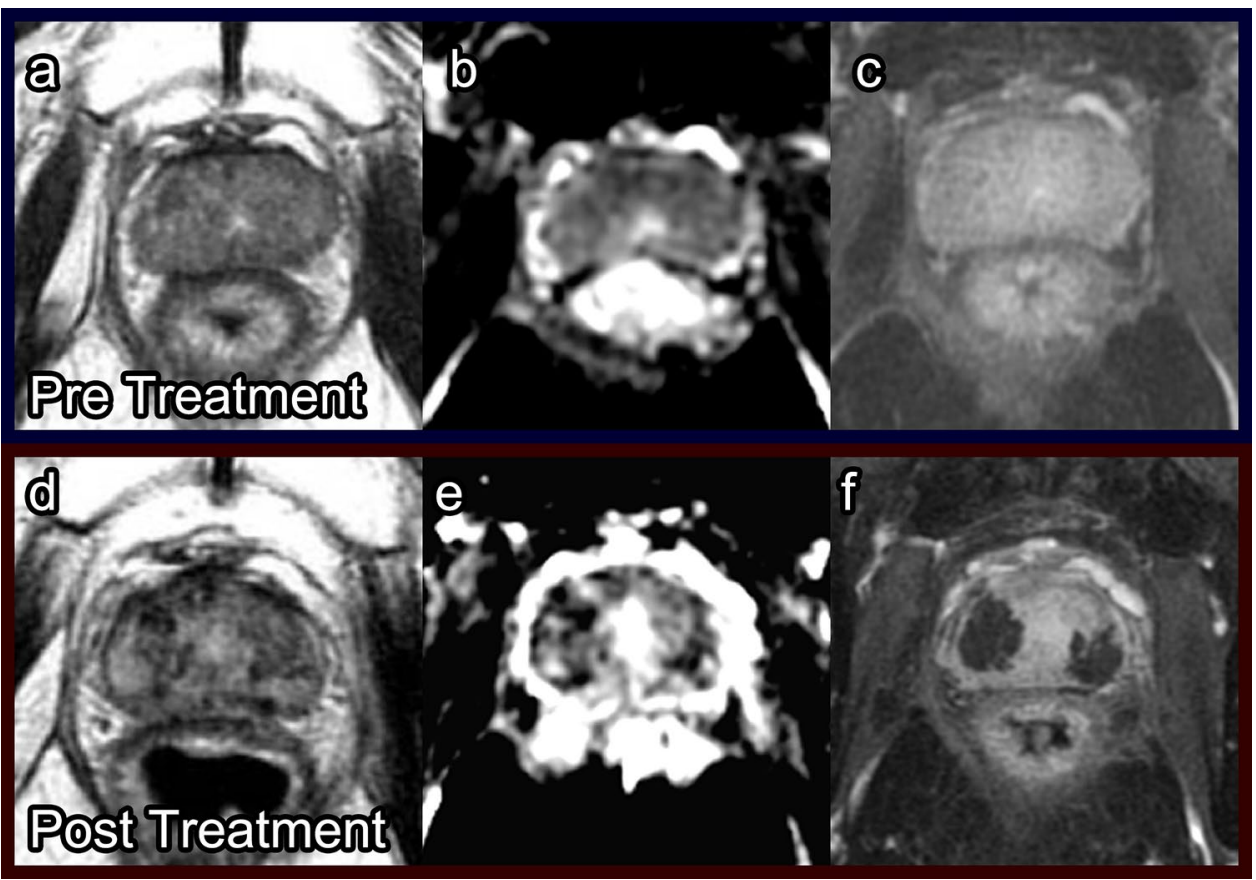

Fig. 9 Low-grade recurrence after photodynamic therapy for recurrent prostate cancer after external beam radiation therapy. Pre-treatment prostate MRI: Fat-suppressed DCE during wash-in in the first 30s post-contrast injection $(\mathbf{a}, \mathbf{b})$ showed focal enhancement indicative of tumor in the left mid and apical posterior medial and lateral peripheral zone (arrow). Biopsy in this region showed Gleason score $4+3$ prostate cancer in $20 \%$ and $30 \%$ of cores. Bilateral laser fibers were placed in the prostate and activated after injection of WST09 (TOOKAD) as part of a research protocol [23]. Post photodynamic therapy prostate MRI at 7 days: DCE (c, d) showed bilateral loss of enhancement in the posterior peripheral zone. There were a few tiny spots of residual enhancement (arrow) in the tumor region. Post photodynamic therapy prostate MRI at 6 months: DCE $(\mathbf{e}, \mathbf{f})$ showed increased enhancement in the treated areas (arrow). Biopsy showed low-grade cancer only (Gleason score $3+3$ in $20 \%$ and $5 \%$ of the cores) in the treated region

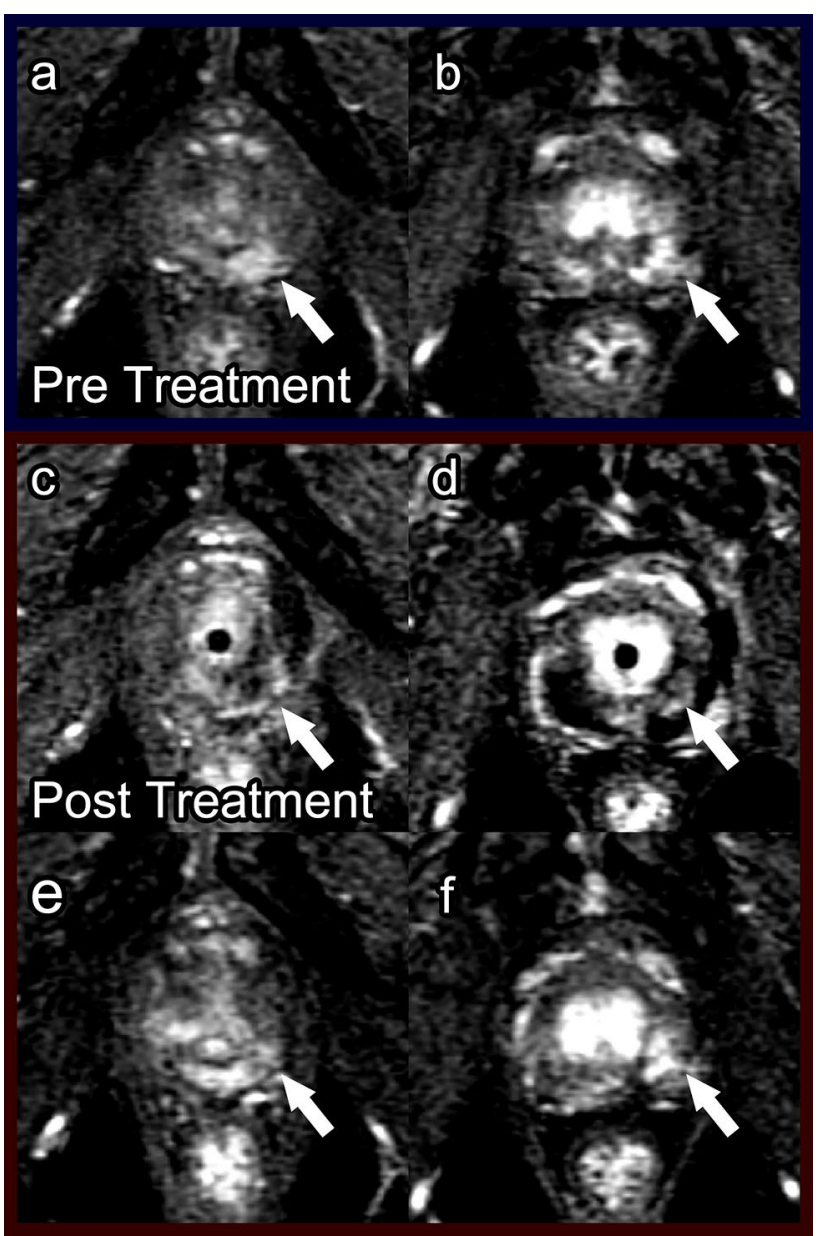




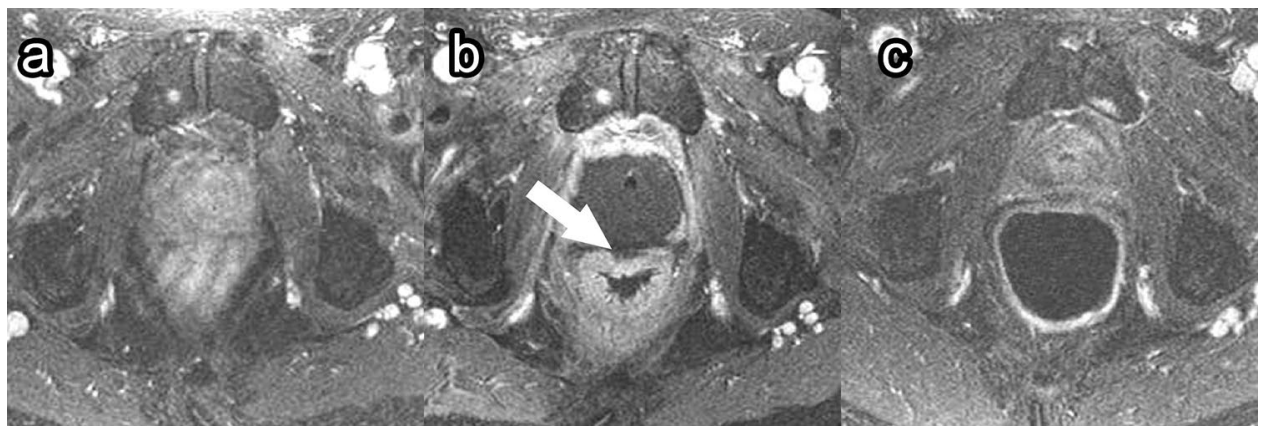

Fig. 10 Rectal wall injury following photodynamic therapy. Pre-treatment axial post-contrast T1 (a), 1-week post-treatment axial postcontrast $\mathrm{T} 1$ (b), and 6-month post-treatment axial post-contrast $\mathrm{T} 1$

PDT, prostate volume is decreased due to scar tissue formation. Additionally, areas of residual necrosis with fluid can be observed, demonstrating low signal on T1WI and high signal on T2WI (Fig. 8).

Follow-up mpMRI imaging has been recommended as soon as 1-week post PDT to evaluate treatment success because this coincides with the time period of maximum tissue necrosis $[23,30]$. Repeat mpMRI should also be done at 6 months post procedure when damage to nontarget tissue is most apparent to establish a baseline. Detection of tumor recurrence is often difficult to assess on T2WI due to the scar signal. ADC map and DCE sequences are the most effective sequences to evaluate recurrence or residual disease (Fig. 9). Residual tumor displays mild diffusion restriction on the ADC map [46]. Any suspicious nodule with early hyperenhancement on DCE or restricted diffusion on DWI should be biopsied.

Complications PDT has low overall complication rates [23, $45,46]$. The most common complication of PDT is extraprostatic necrosis, which is generally limited to the periprostatic fatty tissue but can also include the pubic bone marrow, periurethral tissue, rectal wall (Fig. 10), and muscles close to the prostate $[23,46]$. This extraprostatic necrosis can be seen as a lack of enhancement on post-contrast sequences. Other common complications include hematuria, urinary urgency, and perineal pain [45].

\section{Conclusion}

Novel minimally invasive procedures can effectively treat $\mathrm{BPH}$ and localized prostate cancers. Radiologists reading prostate mpMRI should be familiar with these procedures and the associated complications and normal post-procedure imaging findings to ensure accurate interpretation for evaluation of treatment success and disease recurrence. (c) images demonstrate focal necrosis to the outer prostate wall (b, arrow). At 6-month follow-up (c), the rectal wall has healed, and the prostate has decreased in size due to fibrosis

Funding This study did not receive any funding.

\section{Declarations}

Conflict of interest Francesco Giganti is funded by the UCL Graduate Research Scholarship and the Brahm PhD scholarship in memory of Chris Adams. The other authors declare that they have no conflicts of interest.

Open Access This article is licensed under a Creative Commons Attribution 4.0 International License, which permits use, sharing, adaptation, distribution and reproduction in any medium or format, as long as you give appropriate credit to the original author(s) and the source, provide a link to the Creative Commons licence, and indicate if changes were made. The images or other third party material in this article are included in the article's Creative Commons licence, unless indicated otherwise in a credit line to the material. If material is not included in the article's Creative Commons licence and your intended use is not permitted by statutory regulation or exceeds the permitted use, you will need to obtain permission directly from the copyright holder. To view a copy of this licence, visit http://creativecommons.org/licenses/by/4.0/.

\section{References}

1. Siegel RL, Miller KD, Jemal A (2019) Cancer statistics, 2019. CA Cancer J Clin 69 (1):7-34. https://doi.org/10.3322/caac.21551

2. Hugosson J, Carlsson S (2014) Overdetection in screening for prostate cancer. Curr Opin Urol 24 (3):256-263. https://doi.org/ 10.1097/MOU.0000000000000054

3. Lim KB (2017) Epidemiology of clinical benign prostatic hyperplasia. Asian J Urol 4 (3):148-151. https://doi.org/10.1016/j.ajur. 2017.06.004

4. Pound CR, Partin AW, Eisenberger MA, Chan DW, Pearson JD, Walsh PC (1999) Natural history of progression after PSA elevation following radical prostatectomy. JAMA 281 (17):1591-1597. https://doi.org/10.1001/jama.281.17.1591

5. Bardis MD, Houshyar R, Chang PD, Ushinsky A, Glavis-Bloom J, Chahine C, Bui TL, Rupasinghe M, Filippi CG, Chow DS (2020) Applications of artificial intelligence to prostate multiparametric MRI (mpMRI): Current and emerging trends. Cancers (Basel) 12(5):1204. https://doi.org/10.3390/cancers 12051204

6. de Rooij M, Hamoen EH, Futterer JJ, Barentsz JO, Rovers MM (2014) Accuracy of multiparametric MRI for prostate cancer 
detection: a meta-analysis. AJR Am J Roentgenol 202 (2):343351. https://doi.org/10.2214/AJR.13.11046

7. Daun M, Fardin S, Ushinsky A, Batra S, Nguyentat M, Lee T, Uchio E, Lall C, Houshyar R (2019) PI-RADS Version 2 Is an Excellent Screening Tool for Clinically Significant Prostate Cancer as Designated by the Validated International Society of Urological Pathology Criteria: A Retrospective Analysis. Curr Probl Diagn Radiol. https://doi.org/10.1067/j.cpradiol.2019.06.010

8. Westphalen AC, McCulloch CE, Anaokar JM, Arora S, Barashi NS, Barentsz JO, Bathala TK, Bittencourt LK, Booker MT, Braxton VG, Carroll PR, Casalino DD, Chang SD, Coakley FV, Dhatt R, Eberhardt SC, Foster BR, Froemming AT, Futterer JJ, Ganeshan DM, Gertner MR, Mankowski Gettle L, Ghai S, Gupta RT, Hahn ME, Houshyar R, Kim C, Kim CK, Lall C, Margolis DJA, McRae SE, Oto A, Parsons RB, Patel NU, Pinto PA, Polascik TJ, Spilseth B, Starcevich JB, Tammisetti VS, Taneja SS, Turkbey B, Verma S, Ward JF, Warlick CA, Weinberger AR, Yu J, Zagoria RJ, Rosenkrantz AB (2020) Variability of the positive predictive value of PI-RADS for prostate MRI across 26 centers: experience of the society of abdominal radiology prostate cancer disease-focused panel. Radiology. https://doi.org/10.1148/radiol.2020190646

9. Turkbey B, Rosenkrantz AB, Haider MA, Padhani AR, Villeirs G, Macura KJ, Tempany CM, Choyke PL, Cornud F, Margolis DJ, Thoeny HC, Verma S, Barentsz J, Weinreb JC (2019) Prostate Imaging Reporting and Data System Version 2.1: 2019 Update of Prostate Imaging Reporting and Data System Version 2. Eur Urol 76 (3):340-351. https://doi.org/10.1016/j.eururo.2019.02.033

10. Nguyentat M, Ushinsky A, Miranda-Aguirre A, Uchio E, Lall C, Shirkhoda L, Lee T, Green C, Houshyar R (2018) Validation of Prostate Imaging-Reporting and Data System Version 2: A Retrospective Analysis. Curr Probl Diagn Radiol 47 (6):404-409. https://doi.org/10.1067/j.cpradiol.2017.10.002

11. Nguyen TB, Ushinsky A, Yang A, Nguyentat M, Fardin S, Uchio E, Lall C, Lee T, Houshyar R (2018) Utility of quantitative apparent diffusion coefficient measurements and normalized apparent diffusion coefficient ratios in the diagnosis of clinically significant peripheral zone prostate cancer. Br J Radiol 91 (1088):20180091. https://doi.org/10.1259/bjr.20180091

12. Rampoldi A, Barbosa F, Secco S, Migliorisi C, Galfano A, Prestini G, Harward SH, Di Trapani D, Brambillasca PM, Ruggero V, Solcia M, Carnevale FC, Bocciardi AM (2017) Prostatic Artery Embolization as an Alternative to Indwelling Bladder Catheterization to Manage Benign Prostatic Hyperplasia in Poor Surgical Candidates. Cardiovasc Intervent Radiol 40 (4):530-536. https:// doi.org/10.1007/s00270-017-1582-8

13. Frenk NE, Baroni RH, Carnevale FC, Goncalves OM, Antunes AA, Srougi M, Cerri GG (2014) MRI findings after prostatic artery embolization for treatment of benign hyperplasia. AJR Am J Roentgenol 203 (4):813-821. https://doi.org/10.2214/AJR.13. 11692

14. Zhang H, Shen Y, Pan J, Wang H, Zhong Y, Wang Y, Ye H (2018) MRI features after prostatic artery embolization for the treatment of medium- and large-volume benign hyperplasia. Radiol Med 123 (10):727-734. https://doi.org/10.1007/s11547-018-0904-5

15. de Assis AM, Moreira AM, de Paula Rodrigues VC, Yoshinaga EM, Antunes AA, Harward SH, Srougi M, Carnevale FC (2015) Prostatic artery embolization for treatment of benign prostatic hyperplasia in patients with prostates $>90 \mathrm{~g}$ : a prospective singlecenter study. J Vasc Interv Radiol 26 (1):87-93. https://doi.org/10. 1016/j.jvir.2014.10.012

16. Young S, Golzarian J (2018) Prostatic artery embolization for benign prostatic hyperplasia: a review. Curr Opin Urol 28 (3):284287. https://doi.org/10.1097/MOU.0000000000000495

17. Zhang G, Wang M, Duan F, Yuan K, Li K, Yan J, Chang Z, Wang Y (2015) Radiological Findings of Prostatic Arterial Anatomy for Prostatic Arterial Embolization: Preliminary Study in 55
Chinese Patients with Benign Prostatic Hyperplasia. PLoS One 10 (7):e0132678. https://doi.org/10.1371/journal.pone.0132678

18. Barkin J, Giddens J, Incze P, Casey R, Richardson S, Gange S (2012) UroLift system for relief of prostate obstruction under local anesthesia. Can J Urol 19 (2):6217-6222

19. Garcia C, Chin P, Rashid P, Woo HH (2015) Prostatic urethral lift: A minimally invasive treatment for benign prostatic hyperplasia. Prostate Int 3 (1):1-5. https://doi.org/10.1016/j.prnil.2015.02.002

20. Jones P, Rajkumar GN, Rai BP, Aboumarzouk OM, Cleaveland P, Srirangam SJ, Somani BK (2016) Medium-term Outcomes of Urolift (Minimum 12 Months Follow-up): Evidence From a Systematic Review. Urology 97:20-24. https://doi.org/10.1016/j.urolo gy.2016.05.003

21. Roehrborn CGMD (2016) Prostatic Urethral Lift. Urologic Clinics of North America 43 (3):357-369. https://doi.org/10.1016/j.ucl. 2016.04.008

22. Vargas HA, Akin O, Franiel T, Goldman DA, Udo K, Touijer KA, Reuter VE, Hricak H (2012) Normal central zone of the prostate and central zone involvement by prostate cancer: clinical and MR imaging implications. Radiology 262 (3):894-902. https://doi.org/ 10.1148/radiol.11110663

23. Haider MA, Davidson SR, Kale AV, Weersink RA, Evans AJ, Toi A, Gertner MR, Bogaards A, Wilson BC, Chin JL, Elhilali M, Trachtenberg J (2007) Prostate gland: MR imaging appearance after vascular targeted photodynamic therapy with palladiumbacteriopheophorbide. Radiology 244 (1):196-204. https://doi. org/10.1148/radiol.2441060398

24. Westin C, Chatterjee A, Ku E, Yousuf A, Wang S, Thomas S, Fan X, Eggener S, Karczmar G, Oto A (2018) MRI Findings After MRI-Guided Focal Laser Ablation of Prostate Cancer. AJR Am J Roentgenol 211 (3):595-604. https://doi.org/10.2214/AJR.17. 19201

25. Scheltema MJ, Postema AW, de Bruin DM, Buijs M, Engelbrecht MR, Laguna MP, Wijkstra H, de Reijke TM, de la Rosette J (2017) Irreversible electroporation for the treatment of localized prostate cancer: a summary of imaging findings and treatment feedback. Diagn Interv Radiol 23 (5):365-370. https://doi.org/10.5152/dir. 2017.16608

26. Kirkham AP, Emberton M, Hoh IM, Illing RO, Freeman AA, Allen C (2008) MR imaging of prostate after treatment with highintensity focused ultrasound. Radiology 246 (3):833-844. https:// doi.org/10.1148/radiol.2463062080

27. Kalbhen CL, Hricak H, Shinohara K, Chen M, Parivar F, Kurhanewicz J, Vigneron DB, Carroll PR (1996) Prostate carcinoma: MR imaging findings after cryosurgery. Radiology 198 (3):807-811. https://doi.org/10.1148/radiology.198.3.8628875

28. Elhelf IAS, Albahar H, Shah U, Oto A, Cressman E, Almekkawy M (2018) High intensity focused ultrasound: The fundamentals, clinical applications and research trends. Diagn Interv Imaging 99 (6):349-359. https://doi.org/10.1016/j.diii.2018.03.001

29. Chaussy CG, Thuroff S (2017) High-Intensity Focused Ultrasound for the Treatment of Prostate Cancer: A Review. J Endourol 31 (S1):S30-S37. https://doi.org/10.1089/end.2016.0548

30. Patel P, Mathew MS, Trilisky I, Oto A (2018) Multiparametric MR Imaging of the Prostate after Treatment of Prostate Cancer. Radiographics 38 (2):437-449. https://doi.org/10.1148/rg.20181 70147

31. Notley M, Yu J, Fulcher AS, Turner MA, Cockrell CH, Nguyen D (2015) Pictorial review. Diagnosis of recurrent prostate cancer and its mimics at multiparametric prostate MRI. Br J Radiol 88 (1054):20150362. https://doi.org/10.1259/bjr.20150362

32. Martino P, Scattoni V, Galosi AB, Consonni P, Trombetta C, Palazzo S, Maccagnano C, Liguori G, Valentino M, Battaglia M, Barozzi L (2011) Role of imaging and biopsy to assess local recurrence after definitive treatment for prostate carcinoma (surgery, 
radiotherapy, cryotherapy, HIFU). World J Urol 29 (5):595-605. https://doi.org/10.1007/s00345-011-0687-y

33. Sanda MG, Cadeddu JA, Kirkby E, Chen RC, Crispino T, Fontanarosa J, Freedland SJ, Greene K, Klotz LH, Makarov DV, Nelson JB, Rodrigues G, Sandler HM, Taplin ME, Treadwell JR (2018) Clinically localized prostate cancer: AUA/ASTRO/SUO guideline. Part II: recommended approaches and details of specific care options. J Urol 199 (4):990-997. https://doi.org/10.1016/j.juro. 2018.01.002

34. Bermejo CE, Pisters LL (2003) Cryotherapy for prostate cancer. Expert Rev Anticancer Ther 3 (3):393-401. https://doi.org/10. 1586/14737140.3.3.393

35. Shah TT, Ahmed H, Kanthabalan A, Lau B, Ghei M, Maraj B, Arya M (2014) Focal cryotherapy of localized prostate cancer: a systematic review of the literature. Expert Rev Anticancer Ther 14 (11):1337-1347. https://doi.org/10.1586/14737140.2014.965687

36. Guenther E, Klein N, Zapf S, Weil S, Schlosser C, Rubinsky B, Stehling MK (2019) Prostate cancer treatment with Irreversible Electroporation (IRE): Safety, efficacy and clinical experience in 471 treatments. PLoS One 14 (4):e0215093. https://doi.org/10. 1371/journal.pone.0215093

37. Giganti F, Stabile A, Giona S, Marenco J, Orczyk C, Moore CM, Allen C, Kirkham A, Emberton M, Punwani S (2019) Prostate cancer treated with irreversible electroporation: MRI-based volumetric analysis and oncological outcome. Magn Reson Imaging 58:143-147. https://doi.org/10.1016/j.mri.2019.02.003

38. Beyer LP, Pregler B, Verloh N, Brunn K, Haimerl M, Stroszczynski C, Jung EM, Wiggermann P (2017) Effect of irreversible electroporation of prostate cancer on microcirculation: Imaging findings in contrast-enhanced T1-weighted 3D MRI. Clin Hemorheol Microcirc 67 (3-4):399-405. https://doi.org/10.3233/CH-179220

39. Tay KJ, Amin MB, Ghai S, Jimenez RE, Kench JG, Klotz L, Montironi R, Muto S, Rastinehad AR, Turkbey B, Villers A, Polascik TJ (2019) Surveillance after prostate focal therapy. World J Urol 37 (3):397-407. https://doi.org/10.1007/s00345-018-2363-y

40. van Luijtelaar A, Greenwood BM, Ahmed HU, Barqawi AB, Barret E, Bomers JGR, Brausi MA, Choyke PL, Cooperberg MR, Eggener S, Feller JF, Frauscher F, George AK, Hindley RG, Jenniskens SFM, Klotz L, Kovacs G, Lindner U, Loeb S, Margolis
DJ, Marks LS, May S, McClure TD, Montironi R, Nour SG, Oto A, Polascik TJ, Rastinehad AR, De Reyke TM, Reijnen JS, de la Rosette J, Sedelaar JPM, Sperling DS, Walser EM, Ward JF, Villers A, Ghai S, Futterer JJ (2019) Focal laser ablation as clinical treatment of prostate cancer: report from a Delphi consensus project. World J Urol 37 (10):2147-2153. https://doi.org/10.1007/ s00345-019-02636-7

41. Wenger H, Yousuf A, Oto A, Eggener S (2014) Laser ablation as focal therapy for prostate cancer. Curr Opin Urol 24 (3):236-240. https://doi.org/10.1097/MOU.0000000000000044

42. Natarajan S, Jones TA, Priester AM, Geoghegan R, Lieu P, Delfin M, Felker E, Margolis DJA, Sisk A, Pantuck A, Grundfest W, Marks LS (2017) Focal Laser Ablation of Prostate Cancer: Feasibility of Magnetic Resonance Imaging-Ultrasound Fusion for Guidance. J Urol 198 (4):839-847. https://doi.org/10.1016/j.juro. 2017.04.017

43. Windahl T, Andersson SO, Lofgren L (1990) Photodynamic therapy of localised prostatic cancer. Lancet 336 (8723):1139. https:// doi.org/10.1016/0140-6736(90)92626-s

44. Gheewala T, Skwor T, Munirathinam G (2017) Photosensitizers in prostate cancer therapy. Oncotarget 8 (18):30524-30538. https:// doi.org/10.18632/oncotarget.15496

45. Taneja SS, Bennett J, Coleman J, Grubb R, Andriole G, Reiter RE, Marks L, Azzouzi AR, Emberton M (2016) Final Results of a Phase I/II Multicenter Trial of WST11 Vascular Targeted Photodynamic Therapy for Hemi-Ablation of the Prostate in Men with Unilateral Low Risk Prostate Cancer Performed in the United States. J Urol 196 (4):1096-1104. https://doi.org/10.1016/j.juro. 2016.05.113

46. Kulik M, Nedelcu C, Martin F, Lebdai S, Rousselet MC, Azzouzi AR, Aube C (2014) Post-treatment MRI aspects of photodynamic therapy for prostate cancer. Insights Imaging 5 (6):697-713. https://doi.org/10.1007/s13244-014-0359-8

Publisher's Note Springer Nature remains neutral with regard to jurisdictional claims in published maps and institutional affiliations.

\section{Authors and Affiliations}

\section{Thanh-Lan Bui ${ }^{1}$. Justin Glavis-Bloom ${ }^{1} \cdot$ Chantal Chahine $^{1} \cdot$ Raj Mehta $^{1} \cdot$ Taylor Wolfe $^{1} \cdot$ Param Bhatter ${ }^{1}$. Mark Rupasinghe ${ }^{1}$. Joseph Carbone ${ }^{1} \cdot$ Masoom A. Haider $^{2} \cdot$ Francesco Giganti $^{3,4} \cdot$ Simone Giona ${ }^{5} \cdot$ Aytekin Oto $^{6}$. Grace Lee $^{6} \cdot$ Roozbeh Houshyar ${ }^{1,7}$}

\author{
Thanh-Lan Bui \\ thanhltb@hs.uci.edu \\ Justin Glavis-Bloom \\ jglavisb@hs.uci.edu \\ Chantal Chahine \\ chahinechantal@gmail.com \\ Raj Mehta \\ mehtara@hs.uci.edu \\ Taylor Wolfe \\ twolfe1@hs.uci.edu \\ Param Bhatter \\ pbhatter@hs.uci.edu \\ Mark Rupasinghe \\ mrupasin@hs.uci.edu
}

\author{
Joseph Carbone \\ jacarbon@hs.uci.edu \\ Masoom A. Haider \\ mahaider@radfiler.com \\ Francesco Giganti \\ f.giganti@ucl.ac.uk \\ Simone Giona \\ simone.giona@nhs.net \\ Aytekin Oto \\ aoto@radiology.bsd.uchicago.edu \\ Grace Lee \\ glee@ radiology.bsd.uchicago.edu
}

Department of Radiological Sciences, University of California, Irvine, Orange, CA 92868-3201, USA 
2 Joint Department of Medical Imaging, Sinai Health System, University Health Network, University of Toronto, Toronto, ON, Canada

3 Department of Radiology, University College London Hospital NHS Foundation Trust, London, UK

4 Division of Surgery \& Interventional Science, University College London, London, UK

5 Department of Urology, Frimley Park Hospital, Frimley, Camberley, UK
6 Department of Radiology, University of Chicago, Chicago, IL, USA

7 Department of Radiological Sciences, University of California, Irvine, Bldg. 1 Route 140, Orange, CA 92868-3201, USA 